\title{
Entangled-Pair Transmission Improvement Using Distributed Phase-Sensitive Amplification
}

\begin{abstract}
Anjali Agarwal, James M. Dailey, Paul Toliver, and Nicholas A. Peters
Applied Communication Sciences, 331 Newman Springs Road, Red Bank, New Jersey 07701, USA

(Received 17 April 2014; revised manuscript received 11 October 2014; published 1 December 2014)

We demonstrate the transmission of time-bin entangled photon pairs through a distributed optical phase-sensitive amplifier (OPSA). We utilize four-wave mixing at telecom wavelengths in a 5-km dispersion-shifted fiber OPSA operating in the low-gain limit. Measurements of two-photon interference curves show no statistically significant degradation in the fringe visibility at the output of the OPSA. In addition, coincidence counting rates are higher than direct passive transmission because of constructive interference between amplitudes of input photon pairs and those generated in the OPSA. Our results suggest that application of distributed phase-sensitive amplification to transmission of entangled photon pairs could be highly beneficial towards advancing the rate and scalability of future quantum communications systems.
\end{abstract}

DOI: $10.1103 / P h y s R e v X .4 .041038$

\section{INTRODUCTION}

Photonic entanglement is a critical resource for quantum computing as well as quantum communications, and it can be optimized for transmission through many types of waveguides. However, waveguide propagation loss exponentially reduces quantum communication throughput with increasing transmission distance. In classical optical communications, the problems of loss and the resulting reduced throughputs are solved by optically amplifying the signals. However, amplification of unknown quantum states adds unacceptably high amounts of noise [1]. The added noise reduces quantum communication throughput, which is a function of both state quality and probability of measurement. To circumvent these issues, we utilize known photonic time-bin entangled qubit pairs [2] in combination with a distributed optical phase-sensitive amplifier (OPSA) optimized for the transmission of the entangled pairs. The use of an OPSA provides the potential for significantly better noise performance compared to conventional phaseinsensitive amplifiers [3]. Recent theoretical progress quantified channel-capacity improvements when using OPSAs with coherent state inputs [4]. The benefits of distributed phase-sensitive amplification for classical signals, including coherent states of any amplitude, are well known. It is of great interest to understand how these logarithmic noise advantages can be applied to quantum communications. To that end, we explore experimentally the impact of transmission of entangled photon pairs

\footnotetext{
*aagarwal@appcomsci.com
}

Published by the American Physical Society under the terms of the Creative Commons Attribution 3.0 License. Further distribution of this work must maintain attribution to the author(s) and the published article's title, journal citation, and DOI.
Subject Areas: Photonics, Quantum Physics

through a distributed OPSA. We demonstrate that the throughput relative to direct transmission may be improved when the photons in the entangled pair are simultaneously transmitted down the OPSA. This work suggests that the development of new quantum communication protocols that utilize photon pair encoding and distributed amplification could improve future quantum communication throughputs over direct transmission.

Over the past 20 years, quantum interference between pairs of photons has been a subject of continued interest (see, e.g., Refs. [5-10]). In particular, the application of optical parametric amplification to nonclassical states of light, such as two-photon entangled states, has been considered previously in the demonstration of stimulated emission of entangled photons $[7,8]$, in a theoretical study for the generation of higher photon number N00N states [9] and, more recently, in the characterization of the quantum wave packet [10]. These efforts focused on high-gain amplification of photonic states in nonlinear crystals to bootstrap multiphoton-state creation. In addition, optical parametric amplification has been considered for quantum cloning [11] and for the manipulation $[12,13]$ of continuous-variable (CV) quantum states [14]. Other recent work suggests that OPSAs can be used as preamplifiers in CV quantum key distribution systems to increase detection efficiency [15-17].

The system configuration is comprised of a spontaneous four-wave mixing-based (SFWM) source and a 5-km section of transmission fiber operated as an OPSA. While the source and the OPSA both utilize dispersionshifted fiber, they each operate in different regimes to align with their intended function. First, in contrast to the source, the operation of the 5-km OPSA-based transmission fiber is specifically optimized so as to only offset the intrinsic fiber propagation loss. Second, while the source creates photon pairs from vacuum inputs, the $5-\mathrm{km}$ section of transmission 
fiber is seeded with nondegenerate wavelength signal and idler photon pairs. Injection of signal, idler, and pump wavelengths is required to realize a nondegenerate $\chi^{(3)}$ based OPSA [18]. A final distinction between the operation of the source and the channel is a key fundamental characteristic of an OPSA: The output intensity depends upon the relative phases of the inputs. While a source and an OPSA can both create photon pairs, under the aforementioned input conditions, we discuss how the OPSA exhibits an interference effect that can result in either amplification or deamplification of input pairs below the level of intrinsic fiber loss. Deamplification of the input results from destructive interference between the input photon pairs and those generated within the OPSA, and it is fundamentally different from reducing the pair production efficiency of a source by changing the pumping conditions and hence the resulting phase-matching conditions. While deamplification is not the operating condition, it is observed to confirm that the channel is, in fact, operating as an OPSA instead of a SFWM source. To understand the underlying physical principle, we present a theoretical description of the operation of OPSAs with quantum states consisting of a superposition of vacuum and correlated single-photon pairs. The analysis indicates that our experimental observations are due to constructive interference within the OPSA between the amplitudes of input pairs and internally generated pairs in the OPSA. This interference thus occurs only when the OPSA is seeded with input photon pairs.

We show experimentally, for the first time, that OPSAs can be applied to preserve both the entanglement throughput and the entanglement quality as measured by the two-photon interference (TPI) visibility. Preliminary experimental results of our OPSA-based concepts were first discussed in Ref. [19]. This paper extends upon our initial results by providing a comprehensive description that includes a theoretical and an experimental study of the transmission of photon pairs through a distributed OPSA.

\section{EXPERIMENTAL SETUP}

The experimental setup for transmission of time-bin entanglement $[2,20,21]$ over a distributed OPSA-based fiber transmission channel is shown in Fig. 1. Using spontaneous four-wave mixing, time-bin entanglement is generated in a dispersion shifted fiber (DSF) [21]. The DSF is pumped by picosecond pulses from a tunable modelocked laser (MLL) centered at $1549.4 \mathrm{~nm}$ with a $47-\mathrm{MHz}$ repetition rate. The pump wavelength is chosen to optimize the phase-matching conditions in the dispersion-shifted fiber, which maximizes the four-wave mixing efficiencies in the entangled source and the OPSA. Pump pulses from the MLL are input to a Faraday-mirror-based fiberMichelson interferometer to generate pairs of pump pulses separated by $5 \mathrm{~ns}$. These pulses pump a 136-m-long DSF to produce time-bin entangled signal-idler photon pairs through spontaneous four-wave mixing. The peak pump pulse power is approximately $0.2 \mathrm{~W}$, which generates entangled pairs with a probability of $\approx 2 \times 10^{-3}$ per time bin. This relatively small likelihood is chosen so that the probabilities of generating signal-idler pairs in both time bins simultaneously and multiple-pair generation are negligible. The signal and idler photons are chosen to be $\pm 400 \mathrm{GHz}( \pm 3.2 \mathrm{~nm})$ on either side of the central pump wavelength.

All three optical signals pass through an optical filter (Finisar Waveshaper 4000S), which can independently alter the amplitude and phase of the pump, signal, and idler wavelengths. The outputs from this filter are injected into the distributed OPSA-based transmission link, which consists of a 5-km length of DSF having a zero-dispersion wavelength (ZDW) close to the pump wavelength for optimal phase matching. As we demonstrate below, the output coincidence counting rates depend upon the relative phase between the signal, the idler, and the pump, consistent with the defining feature of an OPSA.

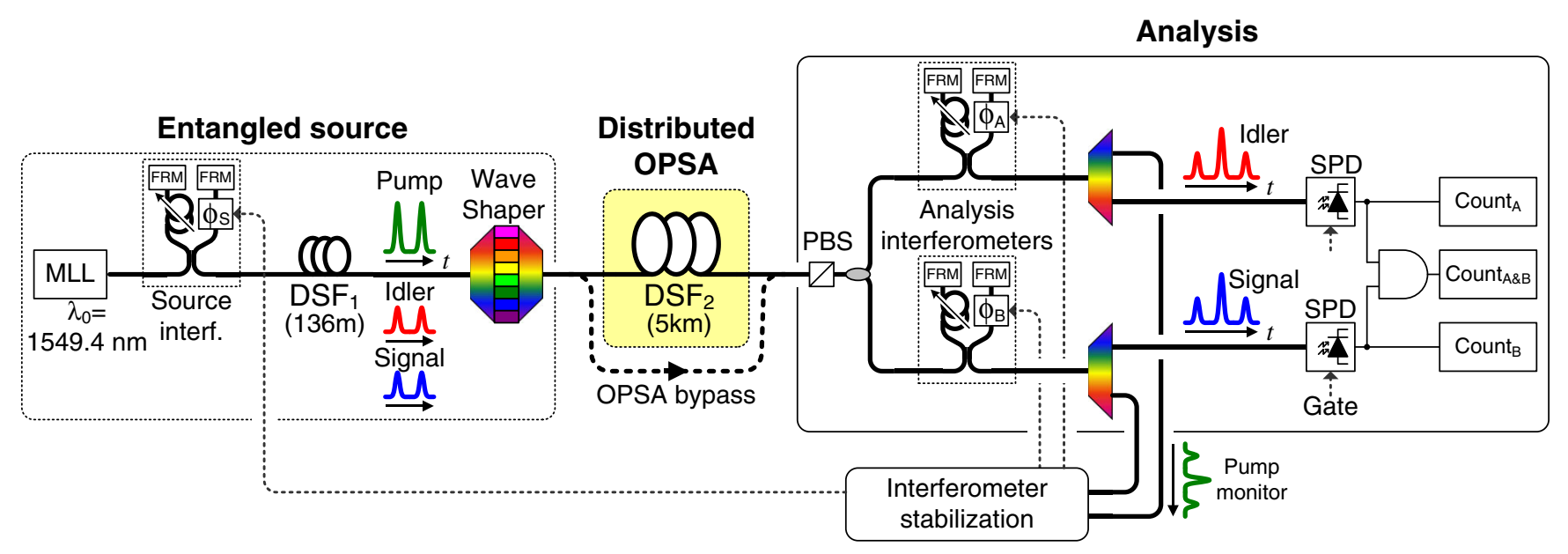

FIG. 1. Experimental setup. We show the mode-locked laser, time bin interferometers, dispersion-shifted fiber, Waveshaper, optical phase sensitive amplifier, polarization beam splitter, and single-photon detectors. 
After the OPSA, a polarizing beam splitter (PBS) filters out the cross-polarized spontaneous Raman noise, and an optical splitter sends the signal and idler photons to their respective analysis time-bin interferometers (TBIs). Out-ofband noise near the signal and idler photons is removed prior to detection (with Princeton Lightwave PGA-600HSX detectors) using thin-film filters with $0.5-\mathrm{dB}$ and $3-\mathrm{dB}$ bandwidths of approximately $0.9 \mathrm{~nm}$ and $1.1 \mathrm{~nm}$, respectively. The two analysis interferometers are used to perform TPI coincidence counting experiments to characterize the entangled pairs before and after transmission through the distributed OPSA.

We actively stabilize the three interferometers to compensate for thermal drifts, which would otherwise result in unstable counting rates. In order to measure stable coincidence counting rates $\left(R_{\mathrm{CC}}\right)$, the analysis TBI phases $(\alpha, \beta)$ must be aligned with the source TBI phase $(\phi)$, as shown in the following relation: $R_{\mathrm{CC}} \approx \frac{1}{2}[1-\cos (\alpha+\beta-2 \phi)]$ [21]. $R_{\mathrm{CC}}$ is maintained by keeping the cosine argument constant. To do this, we take advantage of the classical-level pump signal emerging from the analysis TBIs after transmission through the OPSA. Using a pump phase-dither method, each analysis TBI has its phase locked with the source TBI, and $R_{\mathrm{CC}}$ is maintained at a constant amplitude via automatic feedback to fiber-based phase shifters in the interferometers [22]. In this way, all the interferometers can be continuously stabilized, and they do not require cycling between measurement and stabilization periods.

To measure TPI and verify entanglement quality, we scan a relative phase in each analysis interferometer by adjusting a tunable free-space optical path delay in one interferometer arm by small increments. The resulting delay is short relative to both the detector gate window and the pulse width, so it does not appreciably impact the measured visibility. Each optical delay setting in the analysis TBIs allows us to measure one point on the TPI fringe (see Fig. 3). The TPI fringe is measured by scanning the signal interferometer delay line over a 3-ps range while the idler TBI is unchanged. In addition, we measure a second nonorthogonal basis by adjusting the idler interferometer delay line for a phase shift of one-quarter of one TPI fringe period, and by rescanning the signal TBI.

\section{MEASUREMENT RESULTS}

\section{A. Phase-sensitive amplification of correlated photon pairs}

Before we discuss the TPI results, we describe measurements that are made to characterize the OPSA and confirm its phase-sensitive operation. We characterize the output of the OPSA as a function of the input pump phase relative to that of the signal-idler photon pairs. For the following measurements, the signal and idler TBIs are removed from the setup to reduce lumped loss and maintain higher counting rates. Figure 2 shows the measured coincidence

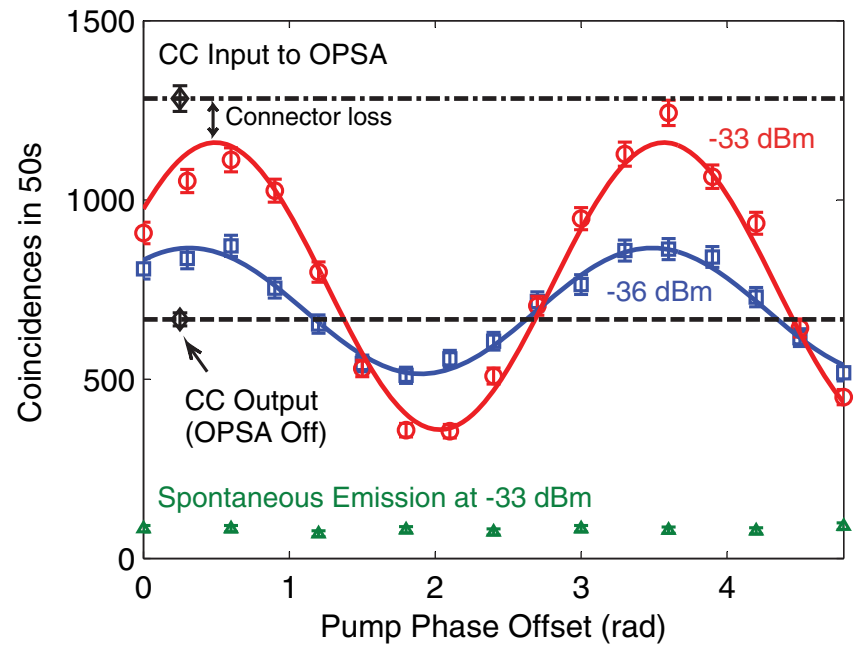

FIG. 2. Measured coincidence counts (CC) in $50 \mathrm{~s}$ for two average pump powers as a function of OPSA pump phase [blue open squares $(-36 \mathrm{dBm})$ and red open circles $(-33 \mathrm{dBm})]$. Spontaneous pairs from the OPSA as a function of pump phase (green triangles). The dashed line corresponds to the coincidence counting rate when the pump is off and to the case of direct fiber transmission. The dash-dotted line shows the coincidence counting rate at the input to the OPSA.

counting rates per a 50 -second measurement interval in a single time bin. The input intensity as measured by coincidence counting rates to the distributed OPSA (dash-dotted line) and the coincidence counting rates after transmission through the 5-km fiber with the pump turned off (dashed line) are shown in Fig. 2. The coincidence counting rates when the OPSA pump is off correspond to simultaneous direct transmission of the entangled pairs. The classically measured loss of the $5-\mathrm{km}$ DSF is $1.5 \mathrm{~dB}$, of which approximately $0.2 \mathrm{~dB}$ is due to connector loss. As the channel loss acts independently on the signal and idler photons, we expect and observe a combined 3-dB drop in coincidence counting rates after transmission through the DSF.

Next, we consider the case where the OPSA pump is turned on. Two sets of data are shown in Fig. 2, corresponding to different average pump powers. The markers are experimental data, while the solid lines are sinusoidal fits to the data. The blue squares correspond to coincidence counting rates when the total average power for the doublepulse pump is $-36 \mathrm{dBm}$, while the red circles are for an average pump power of $-33 \mathrm{dBm}$. As expected, higher (lower) pump powers correspond to higher (lower) coincidence counting rates at the OPSA output. As the pump phase is adjusted relative to the signal and idler, both amplification and deamplification of coincidence counting rates are observed, which confirms the phase-sensitive gain mechanism in the OPSA. At the pump phase yielding maximum amplification, the coincidence counting rates approach the levels measured at the input to the OPSA, with the exception of the bulk connector loss, for which we did not attempt to compensate. 
To rule out the possibility that the enhanced coincidence rate is simply due to the incoherent addition of spontaneous noise from the OPSA, we characterize the spontaneous generation rate in the 5-km distributed OPSA by blocking the input signal and idler photon pairs using the Waveshaper. The measured coincidence counting rates at the OPSA output then give an upper bound on the spontaneous pair contribution to the measurements. The measured spontaneous pairs generated in the OPSA are plotted in Fig. 2 (green open triangles) as a function of pump phase for the pump power, which compensates fiber loss $(-33 \mathrm{dBm})$. The spontaneous coincidence counting rates are insensitive to the OPSA pump phase, as expected, and we measure a mean counting rate of $80 \pm 3$ coincidences per 50-s interval. Next, the direct transmission case is measured by blocking the pump and unblocking the input signal and idler pairs. The measured signal-idler coincidences are $667 \pm 18$ per 50 -s interval. Finally, the pump is set back to $-33 \mathrm{dBm}$, and the coincidence counts increase to $1160 \pm 21$ per $50 \mathrm{~s}$. From these measurements, it is clear that the additional counts at the OPSA output cannot be explained by a simple incoherent sum of spontaneous noise and the surviving input signal from direct transmission. In Sec. III C, we discuss the underlying physics explaining our observed results.

We also bound the possibility of multipair stimulated emission in the OPSA despite its small likelihood given the low gain. The impact of stimulated emission is evaluated by measuring multipair events at the OPSA fiber output. To bound the stimulated emission, we insert a $50 / 50$ coupler after the filtered signal at the output of the OPSA and measure coincidence counts between the two output ports of the coupler. The measured signal-signal coincidence counting rates before and after the OPSA transmission channel are comparable at the pump power that compensates for the fiber loss. The signal-idler coincidence counting rates are approximately 75 times greater than the signal-signal coincidence counting rates, both before and after the OPSA channel. From these measurements and the relatively low gain, we conclude that multipair stimulated emission is negligible in the OPSA transmission link.

\section{B. Visibility of entangled photon pairs at OPSA output}

We next characterize the entanglement quality using a conventional TPI measurement and evaluate the fringe visibility from fits to the data. The OPSA is pumped with an average power of $-33 \mathrm{dBm}$, with the phase of the pump (relative to the signal-idler pairs) set to maximize the gain. The analysis time-bin interferometers are inserted before the single-photon detectors, and the TPI fringes are measured by scanning the analysis interferometer delay lines, which corresponds to adjusting the local phase difference between the arms of each Michelson interferometer, as described in Sec. I. Figures 3(a) and 3(b) show the coincidence counts in 100-s counting intervals as a function of the relative delay change in the signal interferometer. The results from two nonorthogonal measurement bases are shown. The singles rates are also shown, and they do not change significantly when adjusting the relative interferometer delays.

The visibility is obtained by fitting the curve $y=$ $A(1-V \cos [2 \pi f t-\delta])$ to the TPI fringe, where $A$ is the amplitude, $V$ is the visibility, $f$ is the frequency, and $\delta$ is the phase. The visibility and error obtained from curve fitting the raw data in Fig. 3(a) (without subtracting accidentals) for the entangled pairs from the source are $83 \% \pm 3 \%$ (solid curve) and $86 \% \pm 5 \%$ (dashed curve). The corresponding data in the two bases after the 5-km distributed OPSA-based transmission link are shown in Fig. 3(b), and the visibility measurements are $83 \% \pm 3 \%$ (solid curve) and $90 \% \pm 4 \%$ (dashed curve). These results indicate comparable entanglement visibility before and after the OPSA, and they suggest that the quantum characteristics of the transmitted qubit pairs are preserved after transmission through the 5-km OPSA link. Note that the visibility values are obtained without subtracting accidentals and are several standard deviations above the approximate $70.7 \%$ threshold to witness entanglement [23]. Moreover, if the OPSA pump
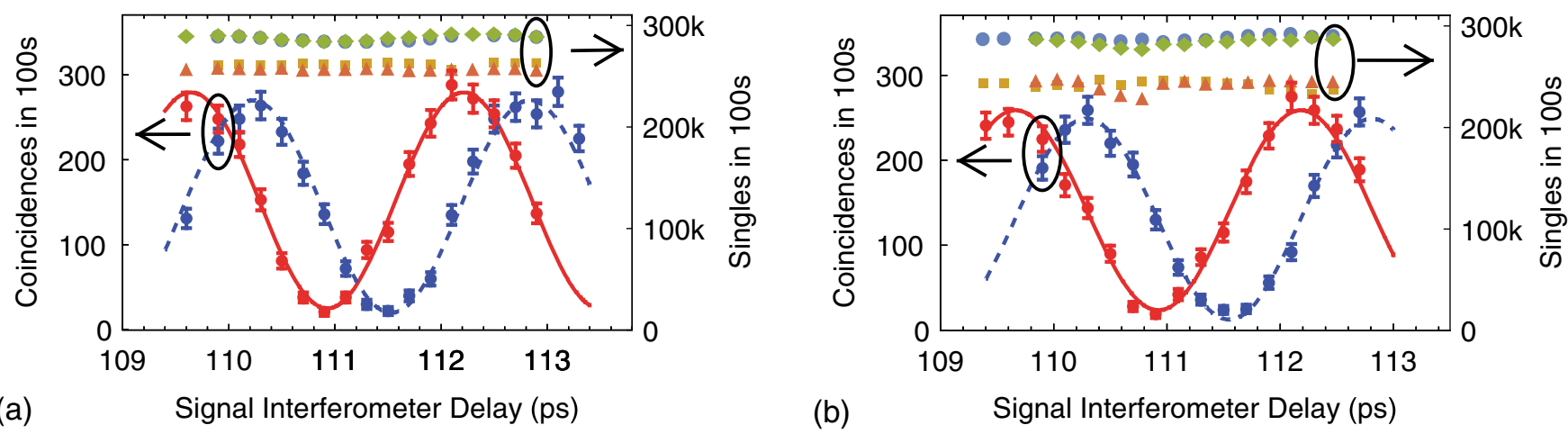

FIG. 3. Two-photon interference fringes in two nonorthogonal measurement bases (a) before and (b) after transmission through a 5-km distributed OPSA transmission link. The corresponding visibilities are obtained from curve fitting the raw data (basis 1 and basis 2 are shown as solid red and dashed blue lines, respectively). The corresponding singles rates are also shown for each measurement basis (right $y$ axis). Basis 1: Blue circles and purple squares. Basis 2: Olive green diamonds and green triangles. 
is turned off, the coincidence rate maximums plotted in Fig. 3(b) are expected to drop by about $3 \mathrm{~dB}$ due to the 1.5-dB single-photon direct transmission fiber loss.

\section{Interference in the OPSA for increased entanglement output}

We now summarize the physical origin of the observed increase in entangled photon-pair counting rates relative to those observed using direct transmission through a passive optical fiber. A detailed derivation is given in the Appendix; here, we summarize and discuss the key results. We assume a configuration that is consistent with our experimental setup: a single-pump, nondegenerate signal and idler, with all wavelengths copolarized. In the experiment, the OPSA is configured to only compensate for fiber loss, and the gain is small. As a result, one OPSA gain parameter $\nu$ is much less than 1 (as described in the Appendix). Ignoring $|\nu|^{3}$ and higher-order terms in $|\nu|$, we rewrite Eq. (A6) to show the phase-sensitive behavior of the output signal-idler correlations:

$$
\begin{aligned}
& \left\langle A_{s}^{\dagger} A_{i}^{\dagger} A_{s} A_{i}\right\rangle \approx e^{-2 \alpha_{1} z}\left\{|\alpha|^{2}|\nu|^{2}+|\beta|^{2}\left[1+11|\nu|^{2}\right]\right. \\
& \left.+\left[\alpha \beta^{*} \mu^{*} \nu+\alpha^{*} \beta \mu \nu^{*}\right]\right\} .
\end{aligned}
$$

Here, $\alpha_{1}$ is the propagation loss, $z$ is the propagation distance, and $\mu$ and $\nu$ are OPSA parameters related to the gain. The input state to the OPSA is $|\psi\rangle=\alpha|00\rangle+\beta|11\rangle$, where $|\alpha|^{2}$ and $|\beta|^{2}$ are the probabilities of vacuum and pair emission from the source, respectively. Note that in our experimental source, $\alpha \gg \beta$.

For our experiment, the exponential loss term in Eq. (1) is $\approx \frac{1}{2}$ and $\mu \approx 1$, so we can further simplify Eq. (1) to

$$
\left\langle A_{s}^{\dagger} A_{i}^{\dagger} A_{s} A_{i}\right\rangle \approx \frac{1}{2}|\alpha \nu|^{2}+|\beta||\alpha \nu| \cos \theta+\frac{1}{2}|\beta|^{2} .
$$

In Eq. (2), we interpret the second term, containing the cosine, as interference between the amplitudes of pairs generated in the OPSA-based channel (related to $\alpha \nu$ ) and the injected entangled photon pairs (with amplitude proportional to $\beta$ ). This interference gives rise to the phase-sensitive behavior observed in Fig. 2. At the optimum phase, this interference is constructive, and the OPSA output significantly exceeds the sum of the input entangled pairs that survive direct transmission $\left(\frac{1}{2}|\beta|^{2}\right)$ and the pairs spontaneously created in the OPSA $\left(\frac{1}{2}|\alpha \nu|^{2}\right)$. This aspect is also clearly demonstrated by the experimental results shown in Fig. 2.

\section{CONCLUSION}

We demonstrate the transmission of entangled photon pairs through a 5-km distributed OPSA. The OPSA operates in the low-gain limit in order to offset the intrinsic fiber transmission loss. As a result, the coincidence counting rate at the output is restored to the input coincidence counting rate, minus a small amount due to bulk connector loss. We present a theoretical analysis for the OPSA transmission channel that illustrates the origin of the increased coincidence counting rates at the OPSA output: constructive interference between the amplitudes of input photon pairs and photon pairs created within the OPSA. Furthermore, using conventional two-photon interference fringe measurements, we observe no significant change in two-photon visibility after transmission through the distributed OPSA.

Quantum communication is highly susceptible to photon losses, and the mitigation of this loss is a critical, yet unsolved, problem. We have considered an approach where the input state is encoded in a way that directly protects against propagation loss by taking advantage of the operating principles of an OPSA. Phase-sensitive amplification based on four-wave mixing requires two-photon input states, which are typically avoided in passive quantum transmission systems because their loss scales as the square of the singlephoton transmission loss. However, as we demonstrate, this loss scaling is greatly mitigated using the OPSA. The OPSA acts collectively on both input photons, and through constructive interference, it adds photon pairs in a way that is related to the state of the input photons.

The input signal, time-bin entanglement between two qubits, is a coherent superposition of quantum states. An entangled pair input is chosen because entanglement is itself the quintessential quantum resource, and we study how it is impacted by transmission through an amplifier. Our results indicate that a two-photon encoding of quantum information and a distributed amplifier combination may help improve the transmission probability of other quantum states. Such an extension may be a promising way to reduce transmission errors, thus enabling distributed quantum computing. While one cannot send any arbitrary state without modifying the OPSA properties, the subspace of states that may be transmitted and that benefit from this approach is large.

The extension of these techniques to improve the transmission of other phase-encoded quantum states is the subject of additional work [24]. We have considered transmission of two phase-encoded nonorthogonal states through a distributed OPSA, where the relative phase between two time bins is encoded. The results reported here foreshadow that a two-photon encoding of quantum information and a distributed amplifier combination may help minimize loss errors in the transmission of additional quantum states. In addition, distributed phase-sensitive amplification may be applied to a variety of qubit systems such as energy-time [25] and polarization entanglement [26-28]. However, this would likely require a different amplifier configuration. For example, if one used polarization qubits, one would need a polarization-independent OPSA configuration (see, for example, Ref. [29]). Finally, fidelity calculations on quantum-level coherent state inputs suggest the benefits provided by using a distributed OPSA scale to longer distances [30]. 
Our results suggest that a properly configured distributed OPSA may enable low-loss high-quality channels for transmission of quantum states encoded onto photon pairs. This low-loss optical channel is a critical component that, when coupled with innovative new protocols, promises to enable new regimes for quantum information transport. Finally, such technology may be useful for creating lowloss optical buffers and quantum memories that could be used in optical quantum computing and communications.

\section{ACKNOWLEDGMENTS}

The authors thank Dr. Colin McKinstrie for many helpful discussions. This material is based upon work supported by DARPAs Defense Sciences Office under Contract No. W31P4Q-13-C-0069. The views and conclusions contained in this document are those of the authors and should not be interpreted as representing official policies, either expressed or implied, of the Defense Advanced Research Projects Agency, the U.S. Army, or the U.S. Government. A. A. and J. M. D. contributed equally to this work.

\section{APPENDIX}

We now provide a quantum mechanical picture of the operation of $\chi^{(3)}$-based OPSAs with a quantum state that consists of a superposition of a vacuum state and a correlated photon pair. The single-pump nondegenerate configuration requires both signal and idler to be present at the input to observe phase-sensitive behavior [18]. For this configuration, the solution to the parametric amplification equations for the output signal and idler field operators, $A_{s}(z)$ and $A_{i}(z)$, respectively, after a distance $z$, is given by (see, for example, Refs. [18,31])

$$
A_{s}(z)=e^{\left(-\alpha_{1} z / 2\right)}\left(\mu A_{s}(0)+\nu A_{i}^{\dagger}(0)\right)
$$

and

$$
A_{i}(z)=e^{\left(-\alpha_{1} z / 2\right)}\left(\mu A_{i}(0)+\nu A_{s}^{\dagger}(0)\right),
$$

where $\mu$ and $\nu$ are given by

$$
\mu=e^{(i / 2)(\Delta \beta+2 \gamma P) z}\left(\cosh (g z)-\frac{i}{2 g} \kappa \sinh (g z)\right)
$$

and

$$
\nu=i e^{(i / 2)(\Delta \beta+2 \gamma P) z} \frac{\gamma P}{g} e^{2 i \theta_{p}} \sinh (g z),
$$

respectively.

$A_{s}\left(A_{i}\right)$ and $A_{s}^{\dagger}\left(A_{i}^{\dagger}\right)$ are the signal (idler) annihilation and creation operators, respectively, so that $A_{s}|n\rangle=\sqrt{n}|n-1\rangle$ and $A_{s}^{\dagger}|n\rangle=\sqrt{n+1}|n+1\rangle$, where $n$ is the number of photons in the input mode. Also, $|\mu|^{2}-|\nu|^{2}=1, \kappa=\Delta \beta-$ $2 \gamma P$ is the phase mismatch, and $g=\sqrt{(\gamma P)^{2}-(\kappa / 2)^{2}}$ is the gain coefficient. $P$ is the pump power, $\gamma$ is the nonlinear coefficient, $\Delta \beta$ is the wave-vector mismatch between the pump, signal, and idler, $\alpha_{1}$ is the propagation loss, and $\theta_{p}$ is the input phase of the pump.

To simplify the analysis, we assume a copolarized pump, signal, and idler; a continuous wave (CW) operation; an undepleted pump; and neglected pump attenuation. The effect of fiber loss on the signal-idler pairs is included; however, the vacuum fluctuations due to the fiber loss are not included because they are negligible with a 5-km OPSA.

We consider the OPSA performance with an input quantum state representative of the output of a lowemission probability entangled pair source that consists of a superposition of a vacuum state and a correlated photon pair and is described by the state $|\psi\rangle=\alpha|00\rangle+\beta|11\rangle$, where $|\alpha|^{2}+|\beta|^{2}=1$. The first term refers to zero photons (vacuum states) in the signal and idler modes with a probability of $|\alpha|^{2}$, while the second term refers to one photon each in the signal and idler modes with a probability of $|\beta|^{2}$. The signal and idler output mean photon number [see Eq. (A5)] and the mean number correlations between the signal and idler photon pairs [see Eq. (A6)] for this input state are given by

$$
\left\langle A_{s}^{\dagger} A_{s}\right\rangle=\left\langle A_{i}^{\dagger} A_{i}\right\rangle=e^{-\alpha_{1} z}\left\{|\alpha \nu+\mu \beta|^{2}+2|\beta \nu|^{2}\right\},
$$

$$
\begin{aligned}
\left\langle A_{s}^{\dagger} A_{i}^{\dagger} A_{s} A_{i}\right\rangle= & e^{-2 \alpha_{1} z}\left\{\left|\mu \nu \alpha+\mu^{2} \beta\right|^{2}+\left|\nu^{2} \alpha+3 \mu \nu \beta\right|^{2}\right. \\
& \left.+4\left|\beta \nu^{2}\right|^{2}\right\} .
\end{aligned}
$$

The input mean photon number in each of the signal and idler modes is $|\beta|^{2}$, and the input mean signal-idler number correlations (as would be counted in coincidence) are given by $|\beta|^{2}$ as well. The gain of the OPSA depends on the values of the OPSA parameters $\mu$ and $\nu$, which in turn depend on the gain coefficient $g$, among other parameters, and may be chosen appropriately to offset the fiber transmission loss.

In the experiment, the OPSA is configured to only compensate for fiber loss and $\nu \ll 1$. As a result, the spontaneous emission in the channel, as well as stimulated emission generating higher photon number states, is expected to be small relative to the input entangled pair rate. This is confirmed experimentally, as discussed in Sec. III A.

[1] S. Fasel, N. Gisin, G. Ribordy, V. Scarani, and H. Zbinden, Quantum Cloning with an Optical Fiber Amplifier, Phys. Rev. Lett. 89, 107901 (2002).

[2] J. Brendel, N. Gisin, W. Tittel, and H. Zbinden, Pulsed Energy-Time Entangled Twin-Photon Source for Quantum Communication, Phys. Rev. Lett. 82, 2594 (1999). 
[3] H. P. Yuen, Reduction of Quantum Fluctuation and Suppression of the Gordon-Haus Effect with Phase-Sensitive Linear Amplifiers, Opt. Lett. 17, 73 (1992).

[4] C. J. McKinstrie, N. Alic, Z. Tong, and M. Karlsson, HigherCapacity Communication Links Based on Two-Mode PhaseSensitive Amplifiers, Opt. Express 19, 11977 (2011).

[5] X. Y. Zou, L. J. Wang, and L. Mandel, Induced Coherence and Indistinguishability in Optical Interference, Phys. Rev. Lett. 67, 318 (1991).

[6] T. J. Herzog, J. G. Rarity, H. Weinfurter, and A. Zeilinger, Frustrated Two-Photon Creation via Interference, Phys. Rev. Lett. 72, 629 (1994).

[7] A. Lamas-Linares, J. C. Howell, and D. Bouwmeester, Stimulated Emission of Polarization-Entangled Photons, Nature (London) 412, 887 (2001).

[8] F. W. Sun, B. H. Liu, Y. X. Gong, Y. F. Huang, Z. Y. Ou, and G. C. Guo, Stimulated Emission as a Result of Multiphoton Interference, Phys. Rev. Lett. 99, 043601 (2007).

[9] R. T. Glasser, H. Cable, J. P. Dowling, F. DeMartini, F. Sciarrino, and C. Vitelli, Entanglement-Seeded-Dual Optical Parametric Amplification: Applications to Quantum Communication, Imaging, and Metrology, Phys. Rev. A 78, 012339 (2008).

[10] R.Z. Vered, Y. Shaked, R. Pomerantz, and A. Pe'er, Observing the Nonclassical Nature of Ultra-broadband Bi-photons at Ultrafast Speed, New J. Phys. 16, 053012 (2014).

[11] R. C. Pooser, A. M. Marino, V. Boyer, K. M. Jones, and P. D. Lett, Low-Noise Amplification of a ContinuousVariable Quantum State, Phys. Rev. Lett. 103, 010501 (2009).

[12] J. Zhang, C. G. Ye, F. Gao, and M. Xiao, Phase-Sensitive Manipulations of Squeezed Vacuum Field in an Optical Parametric Amplifier inside an Optical Cavity, Phys. Rev. Lett. 101, 233602 (2008).

[13] Y. Shang, X. Jia, Y. Shen, C. Xie, and K. Peng, Continuous Variable Entanglement Enhancement and Manipulation by a Sub-threshold Type-II Optical Parametric Amplifier, Opt. Lett. 35, 853 (2010).

[14] C. Weedbrook, S. Pirandola, R. Garcia-Patron, N. J. Cerf, T. C. Ralph, J. H. Shapiro, and S. Lloyd, Gaussian Quantum Information, Rev. Mod. Phys. 84, 621 (2012).

[15] S. Fossier, E. Diamanti, T Debuisschert, R. Tualle-Brouri, and P. Grangier, Improvement of Continuous-Variable Quantum Key Distribution Systems by Using Optical Preamplifiers, J. Phys. B 42, 114014 (2009).

[16] H. Zhang, J. Fang, and G. He, Improving the Performance of the Four-State Continuous-Variable Quantum Key Distribution by Using Optical Amplifiers, Phys. Rev. A 86, 022338 (2012).
[17] Y.-C. Zhang, Z. Li, C. Weedbrook, S. Yu, W. Gu, M. Sun, X. Peng, and H. Guo, Improvement of Two-Way ContinuousVariable Quantum Key Distribution Using Optical Amplifiers, J. Phys. B 47, 035501 (2014).

[18] C. J. McKinstrie and S. Radic, Phase-Sensitive Amplification in a Fiber, Opt. Express 12, 4973 (2004).

[19] J. M. Dailey, A. Agarwal, P. Toliver, and N. A. Peters, Entanglement Transmission through a Distributed Phase Sensitive Amplifier, in Conference on Lasers and Electro Optics, 2014, (Optical Society of America, San Jose, USA, 2014) p. FW1A.5.

[20] I. Marcikic, H. de Riedmatten, W. Tittel, H. Zbinden, M. Legré, and N. Gisin, Distribution of Time-Bin Entangled Qubits Over $50 \mathrm{~km}$ of Optical Fiber, Phys. Rev. Lett. 93, 180502 (2004).

[21] H. Takesue and K. Inoue, Generation of 1.5- $\mu \mathrm{m}$ Band TimeBin Entanglement Using Spontaneous Fiber Four-Wave Mixing and Planar Lightwave Circuit Interferometers, Phys. Rev. A 72, 041804(R) (2005).

[22] P. Toliver, J. M. Dailey, A. Agarwal, and N. A. Peters, Active Stabilization and Continuous Phase Control of Time-Bin Entanglement Interferometers, in Conference on Lasers and Electro Optics, 2014, (Optical Society of America, San Jose, USA, 2014) p. JTu4A.37.

[23] P. G. Kwiat, A. M. Steinberg, and R. Y. Chiao, HighVisibility Interference in a Bell-Inequality Experiment for Energy and Time, Phys. Rev. A 47, R2472 (1993).

[24] J. M. Dailey, A. Agarwal, P. Toliver, and N. A. Peters, Loss Resilience for Two-Qubit State Transmission Using Distributed Phase Sensitive Amplification (work in progress).

[25] J. D. Franson, Bell Inequality for Position and Time, Phys. Rev. Lett. 62, 2205 (1989).

[26] X. Li, P. L. Voss, J. E. Sharping, and P. Kumar, OpticalFiber Source of Polarization-Entangled Photons in the 1550 nm Telecom Band, Phys. Rev. Lett. 94, 053601 (2005).

[27] M. A. Hall, J. B. Altepeter, and P. Kumar, Drop-in Compatible Entanglement for Optical-Fiber Networks, Opt. Express 17, 14558 (2009).

[28] H. Takesue, H. Fukuda, T. Tsuchizawa, T. Watanabe, K. Yamada, Y. Tokura, and S. Itabashi, Generation of Polarization Entangled Photon Pairs Using Silicon Wire Waveguide, Opt. Express 16, 5721 (2008).

[29] K. Inoue, Polarization Independent Wavelength Conversion Using Fiber Four-Wave Mixing with Two Orthogonal Pump Lights of Different Frequencies, J. Lightwave Technol. 12, 1916 (1994).

[30] C. J. McKinstrie, K. Marshall, and C. Weedbrook, Effects of Transmission on Gaussian Optical States (work in progress).

[31] M. Vasilyev, Distributed Phase-Sensitive Amplification, Opt. Express 13, 7563 (2005). 